\title{
ALGEBRAIC METHODS FOR VECTOR BUNDLES ON NON-KÄHLER ELLIPTIC FIBRATIONS
}

\author{
VASILE BRÎNZĂNESCU
}

\begin{abstract}
We survey some parts of the vast literature on holomorphic vector bundles on compact complex manifolds, focusing on the rank-two case vector bundles on non-Kähler elliptic fibrations. It is by no means intended to be a complete overview of this wide topic, but we rather focus on results obtained by the author and his collaborators.
\end{abstract}

\section{INTRODUCTION}

The study of vector bundles over elliptic fibrations has been a very active area of research in both mathematics and physics over the past thirty five years; in fact, there is by now a well understood theory for projective elliptic fibrations (see Donagi [35], [36], Donagi - Pantev [37], Friedman [41], Friedman - Morgan - Witten [42], Bridgeland [12], [13], Bridgeland - Maciocia [14], Bartocci, Bruzzo , Hernandez Ruiperez, Munoz Porras [6], [7], [45], Caldararu [30], [31], et. al.

Not very much is known for non-Kähler elliptic fibrations of complex dimension greater than two. One of the motivations for the study of vector bundles on non-Kähler elliptic 3-folds comes from recent developments in superstring theory, where six-(real)dimensional non-Kähler manifolds occur in string compactifications with non-vanishing background $H$-field (see, for example [8], [44], [33]).

The "space" of our universe is considered to be of the form $\mathbb{R}^{4} \times X$, where $\mathbb{R}^{4}$ is the Minkowski space and $X$ is a complex 3-dimensional manifold of Calabi-Yau type (i.e. $\omega_{X} \cong \mathcal{O}_{X}$ ), not neccessarly Kähler. Thus, the study of moduli spaces of vector bundles over a non-Kähler Calabi-Yau type 3-fold is interesting also for physicists.

The purpose of this paper is to survey some parts of the theory of holomorphic vector bundles on non-Kähler elliptic fibrations, with emphasis on personal contributions.

2010 Mathematics Subject Classification. 32L10, 32J18, 32G13,14J60, 14F05, $13 \mathrm{D} 09$.

Key words and phrases. holomorphic vector bundles, complex compact manifolds, non-Kähler manifolds, elliptic fibrations, moduli spaces.

The author has been partly supported by a research grant of Max Planck Institute for Mathematics in Bonn, March 2016 and by the Fields Institute Focus Program on Topology, Stratified Spaces and Particle Physics, Toronto, August 8$26,2016$. 
The outline of the paper is as follows. In Section 2, we recall first examples and some general results about holomorphic vector bundles over non-algebraic surfaces. Section 3 is devoted to results concerning moduli spaces of rank-two vector bundles on non-Kähler elliptic surfaces. In Section 4, we discuss the moduli spaces of vector bundles on non-Kähler elliptic Calabi-Yau type 3-folds and more generally, on elliptic fibrations which are principal elliptic bundles.

\section{First EXAmples AND FiRst Results}

Let $X$ be a complex manifold. For definitions and the proofs of some of the results see [15]. A holomorphic vector bundle $V$ of rank $r$ over the complex manifold $X$ is called filtrable if there exists a filtration

$$
0=\mathcal{F}_{0} \subset \mathcal{F}_{1} \subset \ldots \subset \mathcal{F}_{r}=V
$$

where $\mathcal{F}_{k}$ is a coherent subsheaf of rank $k$. A holomorphic vector bundle $V$ of rank $r$ over a complex manifold $X$ is called reducible if it admits a coherent analytic subsheaf $\mathcal{F}$ such that

$$
0<\operatorname{rank}(\mathcal{F})<r
$$

and irreducible otherwise. Clearly, for rank 2 non-filtrable is equivalent to irreducible.

Remark 1. Of course, every holomorphic (algebraic) vector bundle $V$ over a projective manifold $X$ is filtrable (since $H^{0}\left(X, V \otimes H^{n}\right)$ is nonzero for big $n$, we obtain a rank one subsheaf of $V$, and so on; here $H$ denotes an ample line bundle on $X$ ), but on non-algebraic manifolds there exist holomorphic vector bundles which are non-filtrable.

The first paper on holomorphic vector bundles in the non-algebraic case was by Elencwajg-Forster (see [40]). In 1982, they constructed in this paper irreducible vector bundles of rank 2 on a complex 2-torus $X$ without divisors (curves); i.e. with Neron-Severi group $N S(X)=$ 0 . This was done by comparing the versal deformation of a filtrable rank 2 vector bundle with the space parametrising extensions producing filtrable rank 2 vector bundles. They proved that, in general, the versal deformation has a bigger dimension, hence it contains also non-filtrable vector bundles.

The next example is due to Schuster, see [58] .

Example 1. Let $X$ be a K3-surface with Picard group Pic $(X)=0$. Then the tangent bundle $T_{X}$ is irreducible.

Since $H^{0}\left(X, T_{X}\right)=0$ it follows easily that the rank 2 vector bundle $T_{X}$ has no coherent analytic subsheaves (see, for example [40] or [15], p. 92). 
The next example is due to Coanda, see [15], p. 104.

Example 2. Let $X$ be a K3-surface with Picard group Pic $(X)=0$. We have an exact sequence of holomorphic vector bundles over $X$

$$
0 \rightarrow \mathcal{O}_{X} \rightarrow T_{X} \oplus T_{X} \rightarrow S^{2} T_{X} \rightarrow 0 .
$$

Then, $S^{2} T_{X}$ is an irreducible holomorphic vector bundle of rank 3.

It is worth to mention here the following results, where holomorphic vector bundles play a key role.

In 1982, Schuster, in [58], proved that for any compact complex surface $X$, every coherent sheaf $F$ on $X$ has global resolutions with locally free sheaves (vector bundles). One of the main steps in the proof was to use rank 2 holomorphic vector bundles from the versal deformation of $T_{X}$.

A long standing problem for compact complex manifolds was to decide if every coherent sheaf has global rsolutions with locally free sheaves (i.e. with vector bundles). A negative answer came only after 20 years. In 2002, C. Voisin proved that this is false for some Kähler compact complex manifolds of dimension $\geq 3$, see [62]. In 2012, Vuletescu gave some new examples of non-Kähler compact complex manifolds of dimension 3 and coherent sheaves $F$ on $X$ having no global resolution by vector bundles, see [66]. The proof that these sheaves do not admit a locally free resolution is very different from Voisin's argument. The manifold $X$ is a Calabi-Eckmann manifold i.e. a principal elliptic bundle over the base $P^{1}(\mathbb{C}) \times P^{1}(\mathbb{C})$, which is diffeomorphic to the product of two real spheres of dimension 3 .

The next paper on holomorphic vector bundles over non-algebraic complex surfaces was by Brinzanescu-Flondor in 1985; see [20]. Let $X$ be a non-algebraic surface, let $V$ be a rank 2 holomorphic vector bundle on $X$ and, let $c_{1}(V), c_{2}(V)$ be the Chern classes of the vector bundle $V$. We have the following result:

Proposition 1. Let $X$ be a non-algebraic surface and let $a \in N S(X)$ be fixed. Then, for every holomorphic rank 2 vector bundle $V$ over $X$ with $c_{1}(V)=a$, we have

$$
c_{2}(V) \geq \min \left\{a^{2} / 4,2 \chi\left(\mathcal{O}_{X}\right)+\left(c_{1}(X) \cdot a+a^{2}\right) / 2\right\} .
$$

In the same paper, [20], one defined a bound for a holomorphic rank 2 vector bundle on a non-algebraic surface with fixed Chern class $a \in N S(X)$ to be filtrable. In another paper, see [21], one gives the range of Chern classes $c_{1}, c_{2}$ of simple filtrable rank two holomorphic vector bundles over complex surfaces $X$ without divisors. These results were extended later by Toma in [60] to the case of complex surfaces of algebraic dimension zero. 
The results of the paper [20] were extended by Banica - Le Potier in 1987, see [4], to the case of holomorphic vector bundles of any rank over a non-algebraic surface.

We need some notations. The Chern classes and the rank can be defined for any analytic coherent sheaf $\mathcal{F}$ over $X$. If $\mathcal{F}$ is locally free, then we have $c_{1}(\mathcal{F})=c_{1}(\operatorname{det}(\mathcal{F})) \in N S(X)$. Generally, by the quoted result of Schuster, see [58], any analytic coherent sheaf $\mathcal{F}$ over a complex surface has a resolution

$$
0 \rightarrow V_{2} \rightarrow V_{1} \rightarrow \mathcal{F} \rightarrow 0
$$

with $V_{i}$ locally free sheaves. Then

$$
c_{1}(\mathcal{F})=\Sigma_{i}(-1)^{i} c_{1}\left(V_{i}\right) \in N S(X) .
$$

Now, let $\mathcal{F}$ be an analytic coherent sheaf over a surface $X$ of rank $r>0$, with Chern classes $c_{1}(\mathcal{F})$ and $c_{2}(\mathcal{F})$. The discriminant $\Delta(\mathcal{F})$ is defined by

$$
\Delta(\mathcal{F}):=\frac{1}{r}\left(c_{2}(\mathcal{F})-\frac{r-1}{2 r} c_{1}^{2}(\mathcal{F})\right) .
$$

The extension of Proposition 1 is given by the following result in [4]:

Theorem 1. Let $X$ be a non-algebraic surface and $\mathcal{F}$ a torsion-free coherent sheaf over $X$ of rank $r$, with Chern classes $c_{1}(\mathcal{F})$ and $c_{2}(\mathcal{F})$. Then $\Delta(\mathcal{F}) \geq 0$.

Other simpler proofs were given later by Brinzanescu in [19] and by Vultescu in [65] (or [15], p. 95).

The converse of this theorem is true for the case $X$ is a primary Kodaira surface; see the paper by Aprodu - Brinzanescu - Toma in 2002, [1].

For a non-algebraic surface $X, a \in N S(X)$ and $r$ a positive integer we can define the following rational positive number

$$
m(r, a):=-\frac{1}{2 r} \max \left\{\Sigma_{1}^{r}\left(a / r-\mu_{i}\right)^{2}, \quad \mu_{i} \in N S(X) \text { with } \Sigma_{1}^{r} \mu_{i}=a\right\} .
$$

Remark 2. When $X$ is a 2-torus and $r=2$ an explicit description of the invariants $m(2, a)$ is given in [20].

The extension of the results for filtrable bundles in [20] is the following result of Banica - Le Potier in [4]:

Theorem 2. A rank $r, r \geq 2$ topological complex vector bundle $V$ over a non-algebraic surface $X$ admits a filtrable holomorphic structure if and only if 


$$
c_{1}(V) \in N S(X) \text { and } \Delta(V) \geq m\left(r, c_{1}(V)\right),
$$

except when $X$ is a K3-surface with $a(X)=0, c_{1}(V) \in r N S(X)$ and $\Delta(V)=\frac{1}{r}$. In this excepted case $V$ admits no holomorphic structures.

There are some other papers on holomorphic vector bundles over non-algebraic manifolds: Braam - Hurtubise [11], Teleman [59], Vuletescu [64], [65], [67].

\section{VECTOR BUNDLES ON NON-KÄHLER ELLIPTIC SURFACES}

Let $X \stackrel{\pi}{\rightarrow} B$ be a minimal non-Kähler elliptic surface with $B$ a smooth curve of genus $g$. It is well-known that $X \stackrel{\pi}{\rightarrow} B$ is a quasibundle over the base $B$, that is, all the smooth fibres are isomorphic to a fixed elliptic curve $E$ and the singular fibres (in a finite number) are multiples of elliptic curves.

Remark 3. For $g=0 X$ is a Hopf surface, for $g=1 X$ is a Kodaira surface and, for $g \geq 2 X$ is called a properly elliptic surface.

Let $E^{*}$ denote the dual of $E$ (we fix a non-canonical identification $E^{*}=P i c^{0}(E)$ by fixing an origin on $\left.E\right)$. The Jacobian surface associated to $X \stackrel{\pi}{\rightarrow} B$ is

$$
J(X)=B \times E^{*} \stackrel{p_{1}}{\rightarrow} B
$$

and $X$ is obtained from the relative Jacobian $J(X)$ by a finite number of logarithmic transformations [51]. We have the following result (see [16], [17], [18]):

Theorem 3. For any minimal non-Kähler elliptic surface we have the isomorphism:

$$
N S(X) / \operatorname{Tors}(N S(X)) \cong \operatorname{Hom}\left(J_{B}, \operatorname{Pic}^{0}(E)\right),
$$

where $N S(X)$ is the Neron-Severi group of the surface and $J_{B}$ denotes the Jacobian variety of the curve $B$.

This result was extended by Brinzanescu - Ueno for torus quasibundles over curves, see [25].

Remark 4. In the case of elliptic surfaces, from the above theorem we get: for any Chern class $c=c_{1}(L)$, with $L \in P i c(X)$ a line bundle, the class $\bar{c} \in N S(X) /$ Tors $(N S(X))$, if it is non-zero, defines a covering map $\bar{c}: B \rightarrow P i c^{0}(E)$, which gives us a section of the Jacobian $J(X)$. This is exactly the spectral curve associated to the line bundle L, defined by Hitchin (see [46]). 
Let $V$ be a holomorphic rank-2 vector bundle on $X$, with fixed $c_{1}(V)=c_{1} \in N S(X)$ and $c_{2}(V)=c_{2} \in \mathbb{Z}$. Now, we fix also the determinant line bundle of $V$, denoted by $\delta=\operatorname{det}(V)$. It defines an involution on the relative Jacobian $J(X)=B \times E^{*}$ of $X$ :

$$
i_{\delta}: J(X) \rightarrow J(X),(b, \lambda) \rightarrow\left(b, \delta_{b} \otimes \lambda^{-1}\right),
$$

where $\delta_{b}$ denotes the restriction of $\delta$ to the fibre $E_{b}=\pi^{-1}(b)$, which has degree zero (see Lemma 2.2 in [22]). Taking the quotient of $J(X)$ by this involution, each fibre of $p_{1}$ becomes $E^{*} / i_{\delta} \cong \mathbb{P}^{1}$ and the quotient $J(X) / i_{\delta}$ is isomorphic to a ruled surface $\mathbb{F}_{\delta}$ over $B$. Let $\eta: J(X) \rightarrow \mathbb{F}_{\delta}$ be the canonical map.

The main existence result of holomorphic rank- 2 vector bundles over non-Kähler elliptic surfaces is the following (see [22]):

Theorem 4. Let $X$ be a minimal non-Kähler elliptic surface over a smooth curve $B$ of genus $g$ and fix a pair $\left(c_{1}, c_{2}\right)$ in $N S(X) \times \mathbb{Z}$. Set $m_{c_{1}}:=m\left(2, c_{1}\right)$ and denote $\bar{c}_{1}$ the class of $c_{1}$ in $N S(X)$ modulo $2 N S(X)$; moreover, let $e_{\bar{c}_{1}}$ be the invariant of the ruled surface $\mathbb{F}_{\bar{c}_{1}}$ determined by $\bar{c}_{1}$. Then, there exists a holomorphic rank-2 vector bundle on $X$ with Chern classes $c_{1}$ and $c_{2}$ if and only if

$$
\Delta\left(2, c_{1}, c_{2}\right) \geq\left(m_{c_{1}}-d_{\bar{c}_{1}} / 2\right),
$$

where $d_{\bar{c}_{1}}:=\left(e_{\bar{c}_{1}}+4 m_{c_{1}}\right) / 2$. Note that both $d_{\bar{c}_{1}}$ and $\left(m_{c_{1}}-d_{\bar{c}_{1}} / 2\right)$ are non-negative numbers. Furthermore, if

$$
\left(m_{c_{1}}-d_{\bar{c}_{1}} / 2\right) \leq \Delta\left(2, c_{1}, c_{2}\right)<m_{c_{1}}
$$

then the corresponding vector bundles are non-filtrable.

Let us suppose for the moment that the minimal non-Kähler elliptic surface $X \stackrel{\pi}{\rightarrow} B$ (which is a quasi-bundle) has no multiple fibres, i.e. it is a principal elliptic bundle. The set of all holomorphic line bundles on $X$ with trivial Chern class is given by the zero component $\operatorname{Pic}^{0}(X)$ of the Picard group Pic $(X)$. By Proposition 1.6 in [59], one has

$$
\operatorname{Pic}^{0}(X) \cong \operatorname{Pic}^{0}(B) \times \mathbb{C}^{*}
$$

and any fibre of $X \stackrel{\pi}{\rightarrow} B$ is $E \cong \mathbb{C}^{*} /<\tau>$, where $<\tau>$ is the multiplicative cyclic group generated by a fixed complex number $\tau$, with $|\tau|>1$. In particular, there exists a universal Poincaré line bundle $\mathcal{U}$ on $X \times P i c^{0}(X)$, whose restriction to

$$
X \times \mathbb{C}^{*}:=X \times\{0\} \times \mathbb{C}^{*} \subset X \times \operatorname{Pic}^{0}(X)
$$


is constructed in terms of constant automorphy factors (for details, see [53] and [22]).

The main tool to study vector bundles on any elliptic surface $X$ is by taking restrictions to the smooth fibres. Note that if $X$ is non-Kähler, then the restriction of any line bundle on $X$ to a smooth fibre of $\pi$ always has degree zero; see [22]. For a rank two vector bundle $V$ over $X$, its restriction to a generic fibre of $\pi$ is semistable; more precisely, its restriction to a fibre $\pi^{-1}(b)$ is unstable on at most an isolated set of points $b \in B$ and, these isolated points are called the jumps of the bundle. Furthermore, there exists a divisor $S_{V}$ in the relative Jacobian $J(X)=B \times E^{*}$ of $X$, called the spectral curve or cover of the bundle, that encodes the isomorphism class of the bundle over each fibre of $\pi$. The spectral curve can be constructed as follows. If the surface $X$ does not have multiple fibres, then there exists a universal bundle $\mathcal{U}$ on $X \times \operatorname{Pic}^{0}(X)$, whose restriction to $X \times \mathbb{C}^{*}$ is also denoted $\mathcal{U}$; we associate to the rank-2 vector bundle $V$ the sheaf on $B \times \mathbb{C}^{*}$ defined by

$$
\tilde{\mathcal{L}}:=R^{1} \pi_{*}\left(S^{*} V \otimes \mathcal{U}\right)
$$

where $s: X \times \mathbb{C}^{*} \rightarrow X$ is the projection onto the first factor, $i d$ is the identity map, and $\pi$ also denotes the projection $\pi:=\pi \times i d$ : $X \times \mathbb{C}^{*} \rightarrow B \times \mathbb{C}^{*}$. This sheaf is supported on a divisor $\tilde{S}_{V}$, defined with multiplicity, that descends to a divisor $S_{V}$ in $J(X)$ of the form

$$
S_{V}:=\left(\sum_{1}^{k}\left\{x_{i}\right\} \times E^{*}\right)+\bar{C},
$$

where $\bar{C}$ is a bisection of $J(X)$ and $x_{1}, x_{2}, \ldots, x_{k}$ are points in $B$ that correspond to the jumps of $V$. The spectral curve of $V$ is defined to be the divisor $S_{V}$. The line bundle $\tilde{\mathcal{L}}$ also descends to a line bundle $\mathcal{L}$ on $J(X)$ (see [22], [23]).

If the fibration $\pi$ has multiple fibres, then one can associate to $X$ a principal $E$-bundle $\pi^{\prime}: X^{\prime} \rightarrow B^{\prime}$ over a $m$-cyclic covering $\epsilon: B^{\prime} \rightarrow B$, where the integer $m$ depends on the multiplicites of the singular fibres. The map $\epsilon$ induces natural $m$-cyclic coverings $J\left(X^{\prime}\right) \rightarrow J(X)$ and $\psi$ : $X^{\prime} \rightarrow X$. By replacing $X$ with $X^{\prime}$ (which does not have multiple fibres) in the above construction, we obtain the spectral cover $S_{\psi^{*} V}$ of the vector bundle $\pi^{*} V$ as a divisor in $J\left(X^{\prime}\right)$. Then, we define the spectral cover $S_{V}$ of $V$ to be the projection of $S_{\psi^{*} V}$ in $J(X)$. This construction led to a natural definition of a twisted Fourier-Mukai transform $\Phi$ for locally free sheaves on $X$, in particular, $\Phi(V)=\mathcal{L}$. For more details, see [23], section 3 and Theorem 3.1.

The determinant line bundle $\delta=\operatorname{det}(V)$ defines the following involution on $J(X)$ : 


$$
i_{\delta}: B \times E^{*} \rightarrow B \times E^{*}, i_{\delta}(b, \lambda)=\left(b, \delta_{b} \otimes \lambda^{-1}\right),
$$

where $\delta_{b}$ denotes the restriction of $\delta$ to the fibre $E_{b}=\pi^{-1}(b)$. The spectral curve $S_{V}$ of $V$ is invariant with respect to this involution. The quotient of $J(X)=B \times E^{*}$ by the involution is a ruled surface $\mathbb{F}_{\delta}:=J(X) / i_{\delta}$ over $B$. Let $\eta: J(X) \rightarrow \mathbb{F}_{\delta}$ be the canonical map. By construction, the spectral curve $S_{V}$ of the bundle $V$ descends to the quotient $\mathbb{F}_{\delta}$; in fact, it is a pullback via $\eta$ of a divisor on $\mathbb{F}_{\delta}$ of the form

$$
\mathcal{G}_{V}:=\Sigma_{1}^{k} f_{i}+A
$$

where $f_{i}$ is the fibre of the ruled surface over the point $x_{i}$ and $A$ is a section of the ruling such that $\eta^{*} A=\bar{C}$. The divisor $\mathcal{G}_{V}$ is called the graph of $V$.

The degree of a vector bundle can be defined on any compact complex manifold $M$ and let $d=\operatorname{dim}_{\mathbb{C}} M$. A theorem of Gauduchon's [43] states that any hermitian metric on $M$ is conformally equivalent to a metric (called now a Gauduchon metric), whose associated (1,1)-form $\omega$ satisfies $\partial \bar{\partial} \omega^{d-1}=0$. Suppose that $M$ is endowed with such a metric and let $L$ be a holomorphic line bundle on $M$. The degree of $L$ with respect to $\omega$ is defined (see [28]), up to a constant factor, by

$$
\operatorname{deg}(L):=\int_{M} F \wedge \omega^{d-1}
$$

where $F$ is the curvature of a hermitian connection on $L$, compatible with $\bar{\partial}_{L}$. Any two such forms differ by an exact $\partial \bar{\partial}$ - exact form. Since $\partial \bar{\partial} \omega^{d-1}=0$, the degree is independent of the choice of connection and is therefore well-defined. This degree is an extension of that in the Kähler case, where we get the usual topological degree. In general, this degree is not a topological invariant, for it can take values in a continuum.

Having defined the degree of holomorphic line bundles, we define the degree of a torsion-free coherent sheaf $\mathcal{V}$ by $\operatorname{deg}(\mathcal{V}):=\operatorname{deg}(\operatorname{det} \mathcal{V})$, where $\operatorname{det} \mathcal{V}$ is the determinant line bundle of $\mathcal{V}$, and the slope of $\mathcal{V}$ by

$$
\mu(\mathcal{V}):=\operatorname{deg}(\mathcal{V}) / \operatorname{rank}(\mathcal{V})
$$

Now, we define the notion of stability: A torsion-free coherent sheaf $\mathcal{V}$ on $M$ is stable if and only if for every coherent subsheaf $\mathcal{S} \subset \mathcal{V}$ with $0<r k(\mathcal{S})<r k(\mathcal{V})$, we have $\mu(\mathcal{S})<\mu(\mathcal{V})$.

Fix a rank-2 vector bundle $V$ on a minimal non-Kähler elliptic surface $X$ and let $\delta$ be its determinant line bundle; there exists a sufficient condition on the spectral cover of $V$ that ensures its stability (see [24]): 
Proposition 2. Suppose that the spectral cover of $V$ includes an irreducible bisection $\bar{C}$ of $J(X)$. Then $V$ is stable.

Remark 5. In fact, in this case, the vector bundle $V$ is irreducible.

Let $X$ be a minimal non-Kähler elliptic surface and consider a pair $\left(c_{1}, c_{2}\right)$ in $N S(X) \times \mathbb{Z}$. For a fixed line bundle $\delta$ on $X$ with $c_{1}(\delta)=$ $c_{1}$, let $\mathcal{M}_{\delta, c_{2}}$ be the moduli space of stable holomorphic rank-2 vector bundles with invariants $\operatorname{det}(V)=\delta$ and $c_{2}(V)=c_{2}$. Note that, for any $c_{1} \in N S(X)$, one can choose a line bundle $\delta$ on $X$ such that

$$
c_{1}(\delta) \in c_{1}+2 N S(X) \text { and } m\left(2, c_{1}\right)=-\frac{1}{2}\left(c_{1}(\delta) / 2\right)^{2} ;
$$

moreover, if there exist line bundles $a$ and $\delta^{\prime}$ such that $\delta=a^{2} \delta^{\prime}$, then there is a natural isomorphism between the moduli spaces $\mathcal{M}_{\delta, c_{2}} \mathcal{M}_{\delta^{\prime}, c_{2}}$, defined by $V \rightarrow a \otimes V$.

This moduli space can be identified, via the Kobayashi-Hitchin correspondence, with the moduli space of gauge-equivalence classes of Hermitian-Einstein connections in the fixed differentiable rank- 2 vector bundle determined by $\delta$ and $c_{2}$ (see, for example, [28], [52]). In particular, if the determinant $\delta$ is the trivial line bundle $\mathcal{O}_{X}$, then there is a one-to-one correspondence between $\mathcal{M}_{\mathcal{O}_{X}, c_{2}}$ and the moduli space of $S U(2)$-instantons, that is, anti-selfdual connections. We can define the map

$$
G: \mathcal{M}_{\delta, c_{2}} \rightarrow \operatorname{Div}\left(\mathbb{F}_{\delta}\right)
$$

that associate to each stable vector bundle its graph in $\operatorname{Div}\left(\mathbb{F}_{\delta}\right)$, called the graph map. In [11], [53], the stability properties of vector bundles on Hopf surfaces were studied by analysing the image and the fibres of this map. In particular, it was shown [53] that the moduli space admits a natural Poisson structure with respect to which the graph map is a Lagrangian fibration whose generic fibre is an abelian variety, i.e. the map $G$ admits an algebraically completely integrable system structure. For general case, the moduli spaces $\mathcal{M}_{\delta, c_{2}}$ are studied by Brinzanescu-Moraru in [24].

We have the following results (see [24]):

Theorem 5. Let $X \stackrel{\pi}{\rightarrow} B$ be a non-Kähler elliptic surface and let $\mathcal{M}_{\delta, c_{2}}$ be defined as above. Then:

(i) There are necessary and sufficient conditions such that $\mathcal{M}_{\delta, c_{2}}$ is nonempty (see Theorem 4).

(ii) If $c_{2}-c_{1}^{2} / 2>g-1$ ( $g$ is the genus of $B$ ), the moduli space $\mathcal{M}_{\delta, c_{2}}$ is smooth on the open dense subset of regular bundles (a regular bundle is a vector bundle for which its restriction to any fibre has its automorphism group of the smallest dimension). 
(iii) If $g \leq 1$, the moduli space $\mathcal{M}_{\delta, c_{2}}$ is smooth of dimension $8 \Delta\left(2, c_{1}, c_{2}\right)$ and $G: \mathcal{M}_{\delta, c_{2}} \rightarrow \operatorname{Div}\left(\mathbb{F}_{\delta}\right)$ is an algebraically completely integrable Hamiltonian system.

(iv) The generic fibre of the graph map $G: \mathcal{M}_{\delta, c_{2}} \rightarrow \operatorname{Div}\left(\mathbb{F}_{\delta}\right)$ is a Prym variety.

(v) Let $\mathbb{P}_{\delta, c_{2}}$ be the set of divisors in $\mathbb{F}_{\delta}$ of the form $\Sigma_{1}^{k} f_{i}+A$, where $A$ is a section of the ruling and the $f_{i}$ 's are fibres of the ruled surface, that are numerically equivalent to $\eta_{*}\left(B_{0}\right)+c_{2} f$. For $c_{2} \geq 2$, the graph map is surjective on $\mathbb{P}_{\delta, c_{2}}$. For $c_{2}<2$, see [24].

(vi) Explicit descriptions of the the singular fibres of $G$ are given, see $[24]$.

Special results on the moduli space $\mathcal{M}_{\delta, c_{2}}$ in the case of primary Kodaira surfaces are given in [2].

\section{VECTOR BUNDLES ON NON-KÄHLER ELLIPTIC FIBRATIONS}

Let $M$ be an n-dimensional compact complex manifold, $T=V / \Lambda$ an m-dimensional complex torus and $X \stackrel{\pi}{\rightarrow} M$ a principal bundle with fiber $T$. The theory of principal torus bundles is developed in great detail in [47]; see also [25]. It is well known that such bundles are described by elements of $H^{1}\left(M, \mathcal{O}_{M}(T)\right)$, where $\mathcal{O}_{M}(T)$ denotes the sheaf of local holomorphic maps from $M$ to $T$. Considering the exact sequence of groups

$$
0 \rightarrow \Lambda \rightarrow V \rightarrow T \rightarrow 0
$$

and taking local sections we obtain the following exact sequence

$$
0 \rightarrow \Lambda \rightarrow \mathcal{O}_{M} \otimes V \rightarrow \mathcal{O}_{M}(T) \rightarrow 0 .
$$

Passing to the cohomology we have the long exact sequence

$$
\begin{aligned}
\cdots \rightarrow H^{1}(M, \Lambda) \rightarrow & H_{M}^{0,1} \otimes V \rightarrow H^{1}\left(M, \mathcal{O}_{M}(T)\right) \stackrel{c^{\mathbb{Z}}}{\rightarrow} \\
& \stackrel{c^{\mathbb{Z}}}{\rightarrow} H^{2}(M, \Lambda) \rightarrow H_{M}^{0,2} \otimes V \rightarrow \cdots
\end{aligned}
$$

By taking the image of the co-cycle defining the bundle via the map $c^{\mathbb{Z}}$ we obtain a characteristic class $c^{\mathbb{Z}}(X) \in H^{2}(M, \Lambda)=H^{2}(M, \mathbb{Z}) \otimes \Lambda$ and also a characteristic class $c(X) \in H^{2}(M, \mathbb{C}) \otimes V$.

Concerning some important sheaves on $X$ we have (see [47]):

$$
\mathcal{K}_{X}=\pi^{*} \mathcal{K}_{M}, \quad R^{i} \pi_{*} \mathcal{O}_{X}=\mathcal{O}_{M} \otimes_{\mathbb{C}} H^{0, i}(T)
$$

and the exact sequence

$$
0 \rightarrow \Omega_{M}^{1} \rightarrow \pi_{*} \Omega_{X}^{1} \rightarrow \mathcal{O}_{M} \otimes_{\mathbb{C}} H^{1,0}(T) \rightarrow 0 .
$$

All the informations concerning the topology of the bundle $X \rightarrow M$ are given by the following invariants

a) The exact sequence (2) gives rise to an element $\gamma \in \operatorname{Ext}^{1}\left(\mathcal{O}_{M} \otimes\right.$ $\left.H^{1,0}(T), \Omega_{M}^{1}\right)=H^{1}\left(\Omega_{M}^{1}\right) \otimes H^{1,0}(T)^{*}$. Thus $\gamma$ is a map $H^{1,0}(T)$ $\rightarrow H^{1,1}(M)$. 
b) The first non-trivial $d_{2}$ - differential in the Leray spectral sequence $\left(d_{2}: E_{2}^{0,1} \rightarrow E_{2}^{2,0}\right)$ of the sheaf $\mathbb{C}_{X}$. We obtain in this way a map $\delta: H^{1}(T, \mathbb{C}) \rightarrow H^{2}(M, \mathbb{C})$. In the same way we may define the maps $\delta^{\mathbb{Z}}: H^{1}(T, \mathbb{Z}) \rightarrow H^{2}(M, \mathbb{Z})$.

c) The first non-trivial $d_{2}$-differential in the Leray spectral sequence of $\mathcal{O}_{X}$, where $d_{2}: H^{0}\left(R^{1} \pi_{*} \mathcal{O}_{X}\right) \rightarrow H^{2}\left(\pi_{*} \mathcal{O}_{X}\right)$. Via the identifications (1) we get a map $\epsilon: H^{0,1}(T) \rightarrow H^{0,2}(M)$.

d) The characteristic classes $c^{\mathbb{Z}}(X)$ and $c(X)$ defined above.

These invariants are related by the following theorem of Höfer (see [47]):

Theorem 6. Let $X \stackrel{\pi}{\rightarrow} M$ be a holomorphic principal T-bundle. Then:

(1) The Borel spectral sequence ([10]) ${ }^{p, q} E_{2}^{s, t}=\sum H^{i, s-i}(M) \otimes$ $H^{p-i, t-p+i}(T)$ degenerates on $E_{3}-$ level and the $d_{2}$-differential is given by $\epsilon$ and $\gamma$.

(2) The Leray spectral sequence $E_{2}^{s, t}=H^{s}(M, \mathbb{C}) \otimes H^{t}(T, \mathbb{C})$ degenerates on $E_{3}-$ level and the $d_{2}-$ differential is given by $\delta$.

(3) Via the identification $H^{1}(T, \mathbb{Z})=\operatorname{Hom}(\Lambda, \mathbb{Z})$ the characteristic class $c^{\mathbb{Z}}$ and the map $\delta^{\mathbb{Z}}$ coincide.

(4) $\delta$ is determined by $\delta^{\mathbb{Z}}$ via scalar extension.

(5) If $H^{2}(M)$ has Hodge decomposition then $\delta$ determines $\epsilon$ and $\gamma$ and conversely.

Firstly, in this section, we shall be concerned with the study of the (coarse) moduli space of line bundles over a principal elliptic bundle $X \rightarrow S$, where $S$ is a compact complex manifold, with fiber $E:=$ $E_{\tau}:=\mathbb{C} / \Lambda(\Lambda=\mathbb{Z} \oplus \tau \mathbb{Z})$. Also we make the assumption that $\delta \neq 0$. In particular, $X \rightarrow S$ does not have the topology of a product. We should note here that if $S$ is Kähler, then X is non-Kähler if and only if $\delta \neq 0$, see [47].

We shall need in the sequel the following result of Deligne, [34], in the formulation of [47], Proposition 5.2.

Theorem 7. Let $X \rightarrow S$ be a principal elliptic bundle. Then the following statements are equivalent:

a) The Leray spectral sequence for $\mathbb{C}_{X}$ degenerates at the $E_{2}$-level;

b) $\delta: H^{1}(E, \mathbb{C}) \rightarrow H^{2}(X, \mathbb{C})$ is the zero map;

c) The restriction map $H^{2}(X, \mathbb{C}) \rightarrow H^{2}(E, \mathbb{C})$ takes a non-zero value in $H_{E}^{1,1}$.

In our case the preceding theorem has a very important consequence

Corollary 1. Let $X \rightarrow S$ be a principal elliptic bundle with $S$ a compact complex manifold and $\delta \neq 0$. Then for any vector bundle $\mathcal{F}$ over $X$ and any $s \in S$ the bundle $\left.\mathcal{F}\right|_{X_{s}}$ has degree 0 . 
Let $X \stackrel{\pi}{\rightarrow} S$ be an elliptic principal bundle with typical fibre an elliptic curve $E_{\tau}$ and base $S$ a smooth manifold. Let $F:(A n / S)^{o p} \rightarrow$ (Sets) be the functor from the category of analytic spaces over $S$ to the category of sets, given, for any commutative diagram

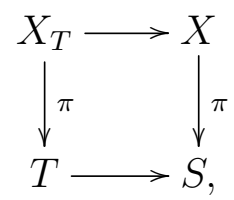

where $X_{T}:=X \times_{S} T$, by

$$
F(T):=\left\{\mathcal{L} \text { invertible on } X_{T} \mid \operatorname{deg}\left(\left.\mathcal{L}\right|_{X_{T, t}}\right)=0, \text { for all } t \in T\right\} / \sim,
$$

where $\mathcal{L}_{1} \sim \mathcal{L}_{2}$ if there is a line bundle $L$ on $T$ such that $\mathcal{L}_{1} \simeq \mathcal{L}_{2} \otimes \pi^{*} L$.

A variety $J$ over $S$ will be called the relative Jacobian of $X$ if

(i) it corepresents the functor $F$, see [49] Definition 2.2.1, i.e. there is a natural transformation $F \stackrel{\sigma}{\rightarrow} \operatorname{Hom}_{S}(-, J)$ and for any other variety $N / S$ with a natural transformation $F \stackrel{\sigma^{\prime}}{\rightarrow} \operatorname{Hom}_{S}(-, N)$ there is a unique $S$-morphism $J \stackrel{\nu}{\rightarrow} N$ such that $\nu_{*} \circ \sigma=\sigma^{\prime}$.

(ii) for any point $s \in S$ the map $F(\{s\}) \rightarrow \operatorname{Hom}_{S}(\{s\}, J) \simeq J_{s}$ is bijective. Then each fibre $J_{s}$ is the Jacobian of the fibre $X_{s} \simeq E$.

If $X$ is projective, the existence of the relative Jacobian is well known, because it can be identified with the coarse relative moduli space of stable locally free sheaves of rank 1 and degree 0 on the fibres of $X$, see [49], [30]. The relative Jacobian exists also in our non-Kähler case. It is just the product $S \times E^{*}$ and has the following special properties (see [26]):

Theorem 8. (i) The functor $F$ is corepresented by $J:=S \times E^{*}$.

(ii) For any point $s \in S$ the map $F(\{s\}) \rightarrow \operatorname{Hom}_{S}(\{s\}, J) \simeq J_{s} \simeq E^{*}$ is bijective.

(iii) The map $\sigma(T)$ is injective for any complex space $T$.

(iv) The functor $F$ is locally representable by $J=S \times E^{*}$, i.e. if $U \subset S$ is a trivializing open subset, $\sigma(U)$ is bijective.

It will follow from Theorem 9 that the relative Jacobian $J=S \times E^{*}$ is only a coarse moduli space under our assumption on $X$. However, by property (iv) of the theorem one can find a system of local universal sheaves which will form a twisted sheaf as in [30], Chapter 4.

In the following we replace the relative Jacobian $J$ by $S \times E$ via the canonical isomorphism between $E$ and $E^{*}$. Then the local trivilalizations $X_{i} \stackrel{\theta_{i}}{\rightarrow} S_{i} \times E$ are at the same time isomorphisms between $X_{i}$ and $J_{i}:=S_{i} \times E$. The local universal sheaves $\mathcal{U}_{i}$ on $X_{i J}=$ $J \times_{S} X_{i}=J_{i} \times_{S_{i}} X_{i}$ are then given as pull backs of the universal sheaf $\mathcal{O}_{E \times E}(\Delta) \otimes p_{2}^{*} \mathcal{O}_{E}\left(-p_{0}\right)$ for the classical Jacobian of the elliptic curve $E$, after fixing an origin $p_{0} \in E$ and where $\Delta$ is the diagonal. 
Denoting by $\rho_{i}$ the composition of maps

$$
X_{i J} \stackrel{i d \times \theta_{i}}{\longrightarrow} J \times_{S}\left(S_{i} \times E\right) \simeq S_{i} \times E \times E \rightarrow E \times E,
$$

and by $p_{X}$ the projection from $X_{i J}$ to $X_{i}$, the local universal sheaf becomes

$$
\mathcal{U}_{i}=\rho_{i}^{*}\left(\mathcal{O}_{E \times E}(\Delta) \otimes p_{2}^{*} \mathcal{O}_{E}\left(-p_{0}\right)\right) \simeq \mathcal{O}_{X i J}\left(\Gamma_{i}\right) \otimes p_{X}^{*} \mathcal{O}_{X_{i}}\left(-s_{i}\right),
$$

where $\Gamma_{i}$ is the inverse of the diagonal (or the graph of the map $\theta_{i}$ ) and $s_{i}$ is the section of $X_{i}$ corresponding to the reference point $p_{0}$ under the isomorphism $\theta_{i}$, see [30], Proposition 4.2.3.

To measure the failure of these bundles to glue to a global universal one let us consider the line bundles $\mathcal{M}_{i j}:=\mathcal{U}_{i} \otimes \mathcal{U}_{j}^{-1}$ over $J \times_{S} X_{i j}$. Then the restriction of $\mathcal{M}_{i j}$ to a fibre $X_{s}$ of the projection $J \times_{s} X_{i} \stackrel{q_{i}}{\rightarrow} J$ is trivial because both $\mathcal{U}_{j}$ and $\mathcal{U}_{i}$ restrict to isomorphic sheaves. It follows that there are invertible sheaves $\mathcal{F}_{i j}$ on $J_{i j}=S_{i j} \times E$ such that $\mathcal{M}_{i j}=q_{i}^{*} \mathcal{F}_{i j}$.

This collection of line bundles satisfies the following properties:

1. $\mathcal{F}_{i i}=\mathcal{O}_{J_{i}}$;

2. $\mathcal{F}_{j i}=\mathcal{F}_{i j}^{-1}$

3. $\mathcal{F}_{i j} \otimes \mathcal{F}_{j k} \otimes \mathcal{F}_{k i}=: \mathcal{F}_{i j k}$ is trivial, with trivialization induced by the canonical one of $\mathcal{M}_{i j} \otimes \mathcal{M}_{j k} \otimes \mathcal{M}_{k i}$;

4. $\mathcal{F}_{i j k} \otimes \mathcal{F}_{j k l}^{-1} \otimes \mathcal{F}_{k l i} \otimes \mathcal{F}_{l i j}^{-1}$ is canonically trivial.

These conditions tell us that the collection $\left\{\mathcal{F}_{i j}\right\}$ represents a gerb (see [37]) and gives rise to an element $\alpha \in H^{2}\left(J, \mathcal{O}_{J}^{*}\right)$. More explicitly, $\alpha$ is defined as follows. We may assume that the sheaves $\mathcal{F}_{i j}$ are already trivial with trivializations $a_{i j}: \mathcal{O}_{J} \simeq \mathcal{F}_{i j}$ over $J_{i j}$.

If $c_{i j k}: \mathcal{O}_{J} \simeq \mathcal{F}_{i j k}$ is the isomorphism which is induced by the canonical trivialization of $\mathcal{M}_{i j} \otimes \mathcal{M}_{j k} \otimes \mathcal{M}_{k i}$, then

$$
a_{i j} \otimes a_{j k} \otimes a_{k i}=\alpha_{i j k} c_{i j k}
$$

with scalar functions $\alpha_{i j k}$ which then define a cocycle for the sheaf $\mathcal{O}_{J}^{*}$, thus defining the class $\alpha \in H^{2}\left(J, \mathcal{O}_{J}^{*}\right)$, see [30], section 4.3. It is straightforward to prove:

Lemma 1. The sheaves $\mathcal{U}_{i}$ can be glued to a global universal sheaf if and only if the class $\alpha=0$.

The element $\alpha$ is related to the element $\xi \in H^{1}\left(S, \mathcal{O}_{S}(E)\right)$ which is defined by the cocycle of the elliptic bundle $X \rightarrow S$, using the Ogg-Shafarevich group $\amalg_{S}(J)$ of $J$, see [30], section 4.4. There is an exact sequence $0 \rightarrow \operatorname{Br}(S) \rightarrow \operatorname{Br}(J) \stackrel{\pi}{\rightarrow} \amalg_{S}(J) \rightarrow 0$, where $\operatorname{Br}(S) \simeq$ $H^{2}\left(J, \mathcal{O}_{J}^{*}\right)$ is the analytic Brauer group of $S$ and $\amalg_{S}(J)$ is isomorphic to $H^{1}\left(S, \mathcal{O}_{S}(E)\right)$ in our setting. We have the following result (see [30], Theorem 4.4.1):

Theorem 9. $\xi=\pi(\alpha)$. 
Because $\xi \neq 0$ in our case, $\alpha \neq 0$, and thus the local universal sheaves cannot be glued to a global universal sheaf by preserving the bundle structure on the elliptic fibres.

The collection of local universal sheaves above can be considered as an $\alpha$-twisted sheaf with which one can define a Fourier-Mukai transform. Recall the definition of an $\alpha$-twisted sheaf on a complex space or on an appropriate scheme $X$. Let $\alpha \in C^{2}\left(\mathfrak{U}, \mathcal{O}_{X}^{*}\right)$ be a Čech 2cocycle, given by an open cover $\mathfrak{U}=\left\{U_{i}\right\}_{i \in I}$ and sections $\alpha_{i j k} \in$ $\Gamma\left(U_{i} \cap U_{j} \cap U_{k}, \mathcal{O}_{X}^{*}\right)$. An $\alpha$-twisted sheaf on $X$ will be a pair of families $\left(\left\{\mathcal{F}_{i}\right\}_{i \in I},\left\{\varphi_{i j}\right\}_{i, j, \in I}\right)$ with $\mathcal{F}_{i}$ a sheaf of $\mathcal{O}_{X}$-modules on $U_{i}$ and $\varphi_{i j}:\left.\left.\mathcal{F}_{j}\right|_{U_{i} \cap U_{j}} \rightarrow \mathcal{F}_{i}\right|_{U_{i} \cap U_{j}}$ isomorphisms such that

- $\varphi_{i i}$ is the identity for all $i \in I$.

- $\varphi_{i j}=\varphi_{j i}^{-1}$, for all $i, j \in I$.

- $\varphi_{i j} \circ \varphi_{j k} \circ \varphi_{k l}$ is multiplication by $\alpha_{i j k}$ on $\left.\mathcal{F}_{i}\right|_{U_{i} \cap U_{j} \cap U_{k}}$ for all $i, j, k \in I$.

It is easy to see that the coherent $\alpha$-twisted sheaves on $\mathrm{X}$ make up an abelian category and thus give rise to a derived category $\mathcal{D}^{b}(X, \alpha)$. For further properties of $\alpha$-twisted sheaves, see [30].

With the notation above, the family $\left(\mathcal{U}_{i}\right)$ becomes a twisted sheaf $\mathcal{U}$ w.r.t. the cocycle $p_{J}^{*} \alpha$ of the sheaf $\mathcal{O}_{J \times_{S} X}^{*}$ as follows. The trivializations $a_{i j}$ of the $\mathcal{F}_{i j}$ induce isomorphisms $\phi_{i j}: \mathcal{U}_{j} \simeq \mathcal{U}_{i}$ which satisfy the definition of a twisted sheaf because of identity 4 . We also need the dual $\mathcal{V}$ of $\mathcal{U}$ on $J \times_{S} X$ which locally over $S_{i}$ is given by

$$
\mathcal{V}_{i}=\rho_{i}^{*}\left(\mathcal{O}_{E \times E}(-\Delta) \otimes p_{2}^{*} \mathcal{O}_{E}\left(p_{0}\right)\right) \simeq \mathcal{O}_{X i J}\left(-\Gamma_{i}\right) \otimes p_{X}^{*} \mathcal{O}_{X_{i}}\left(s_{i}\right)
$$

It follows that $\mathcal{V}_{i}$ is $\alpha^{-1}$-twisted. We let $\mathcal{V}^{0}$ and $\mathcal{U}^{0}$ denote the extensions of $\mathcal{V}$ and $\mathcal{U}$ to $J \times X$ by zero.

The following theorem (see [26]) supplies us with the main tool for the treatment of the moduli spaces $M_{X}(n, 0)$ of relatively semistable vector bundles on $\mathrm{X}$ of rank $n$ and degree 0 on the fibres $X_{s}$ (for vector bundles on elliptic curves see [3], [61]). It is an analog of the Theorem 6.5 .4 [30] (see also [31]):

Theorem 10. Let $X \stackrel{\pi}{\rightarrow} S$ be an elliptic principal fiber bundle. Let $\alpha \in \operatorname{Br}(J)$ be the obstruction to the existence of the universal sheaf on $J \times_{S} X$ and let $\mathcal{U}$ be the associated $p_{J}^{*}(\alpha)$-twisted universal sheaf on $J \times_{S} X$ with its dual $\mathcal{V}$ as above.

Then the twisted Fourier-Mukai transform $\Psi: \mathcal{D}^{b}(J, \alpha) \rightarrow \mathcal{D}^{b}(X)$, given by $\Psi(\mathcal{F}):=R p_{X *}\left(\mathcal{V}^{0} \otimes^{L} L p_{J}{ }^{*} \mathcal{F}\right)$ is an equivalence of categories, where $p_{J}$ and $p_{X}$ are the product projections

$$
J \stackrel{p_{J}}{\longleftarrow} J \times X \stackrel{p_{X}}{\longrightarrow} X
$$

Note here that $\left.\mathcal{V}^{0} \otimes^{L} L p_{J}{ }^{*} \mathcal{F}\right)$ is an object in the category of untwisted sheaves. 
Remark 6. Similar results were obtain in different settings by BenBassat [9] and Burban - Kreussler [29]. Related results were obtain in [32].

In the sequel we shall work with the adjoint transform $\Phi(-)=$ $R p_{J *}\left(\mathcal{U}^{0} \otimes^{L} L p_{X}^{*}(-)\right)$ of $\Psi$ with kernel $\mathcal{U}^{0}$. It is the reverse equivalence, see [14], 8.4, [48], [6] for the untwisted situation.

Now, we shall apply the twisted Fourier-Mukai transform to the moduli problem for rank- $n$ relatively semi-stable vector bundles on the principal elliptic bundle $X$. By Deligne's theorem (Theorem 7), the degree of the restriction $\mathcal{F}_{s}$ of any vector bundle $\mathcal{F}$ on $X$ is 0 for any $s \in S$. Therefore we consider the set $M S_{X}(n, 0)$ of rank- $n$ vector bundles on $X$ which are fibrewise semistable (see [3], [42]) and of degree zero, together with its quotient

$$
M_{X}(n, 0):=M S_{X}(n, 0) / \sim
$$

of equivalence classes, where two bundles are defined to be equivalent if they are fibrewise S-equivalent (for S-equivalence see [49].

We denote by $\Phi^{i}(\mathcal{F})$ the $i$-th term of the complex $\Phi(\mathcal{F})$. We say that the sheaf $\mathcal{F}$ is $\Phi-\mathrm{WIT}_{i}$ (the weak index theorem holds) if $\Phi^{i}(\mathcal{F}) \neq 0$ and $\Phi^{j}(\mathcal{F})=0$ for any $j \neq i$. Moreover if $\mathcal{F}$ is $\operatorname{WIT}_{i}$ and $\Phi^{i}(\mathcal{F})$ is locally free we say that $\mathcal{F}$ is $\mathrm{IT}_{i}$, see [54].

Let $\mathcal{F}$ be a $\mathrm{WIT}_{1}$ sheaf on $X$. The spectral cover $C(\mathcal{F})$ of $\mathcal{F}$ is the 0 -th Fitting subscheme (see [54], [39]) of $J$ given by the Fitting ideal sheaf Fitt $_{0}\left(\Phi^{1}(\mathcal{F})\right)$ of $\Phi^{1}(\mathcal{F})$. For details see [26].

In this way we obtain a map from $M_{X}(n, 0)$ to $S \times \operatorname{Sym}^{n} E$, where $\operatorname{Sym}^{n} E:=E^{n} / \mathfrak{S}_{n}$ is the $n$-th symmetric power of $E$ as the quotient of $E^{n}$ by the symmetric group $\mathfrak{S}_{n}$. Then $S \times \operatorname{Sym}^{n} E$ is a complex manifold of dimension $n+2$ and can be thought of as the relative space of cycles of degree $n$ in $E$. We will show that this map is part of a transformation of functors with target $\operatorname{Hom}_{S}\left(-, S \times \operatorname{Sym}^{n} E\right)$ and that $S \times \operatorname{Sym}^{n} E$ corepresents the moduli functor $\mathcal{M}_{X}(n, 0)$ for $M_{X}(n, 0)$ defined as follows.

For any complex space $T$ over $S$ let the set $\mathcal{M}_{X}(n, 0)(T)$ be defined by

$$
\mathcal{M}_{X}(n, 0)(T):=\mathcal{M S}_{X}(n, 0)(T) / \sim,
$$

where $\mathcal{M S}_{X}(n, 0)(T)$ is the set of vector bundles on $X_{T}$ of rank $n$ and fibre degree 0 , and where the equivalence relation $\mathcal{F} \sim \mathcal{G}$ is defined by $S$-equivalence of the restricted sheaves $\mathcal{F}_{t}$ and $\mathcal{G}_{t}$ on the fibres $X_{T_{t}}$. The functor property is then defined via pull backs.

We are going to describe the spectral cover as a functor below. For that let $T \rightarrow S$ be a complex space over $S$ and let $\Phi_{T}$ be the FourierMukai transform for the product $J_{T} \times X_{T}$ with the pull back $\mathcal{U}_{T}$ of $\mathcal{U}$ as kernel. By [7], Proposion 2.7 and Corollary 2.12, any bundle $\mathcal{F}_{T}$ in $\mathcal{M S}_{X}(n, 0)(T)$ is also $\Phi_{T}-W I T_{1}$ and admits a spectral cover 
$C\left(\mathcal{F}_{T}\right) \subset T \times E$ defined by the Fitting ideal $F i t t_{0} \Phi_{T}^{1}\left(\mathcal{F}_{T}\right)$ (see also $[45])$.

Lemma 2. If $T$ is reduced, then $C\left(\mathcal{F}_{T}\right)$ is flat over $T$.

For the proof, one uses the Douady's flatness criterion [38]; see [26].

Lemma 3. The spectral cover is compatible with base change: For any morhism

$h: T^{\prime} \rightarrow T$ over $S$ and any bundle $\mathcal{F}_{T}$ in $\mathcal{M S}_{X}(n, 0)(T)$,

$$
h^{*} C\left(\mathcal{F}_{T}\right) \simeq C\left(h^{*} \mathcal{F}_{T}\right)
$$

For the proof, see [26].

The spectral covers $C\left(\mathcal{F}_{T}\right)$ lead us to consider the relative Douady functors

$$
\mathcal{D}^{n}:(A n / S)^{o p} \rightarrow(\text { Sets }),
$$

where $(A n / S)$ denotes the category of complex analytic spaces over $S$ and where a set $\mathcal{D}^{n}(T)$ for a morphism $T \rightarrow S$ is defined as the set of analytic subspaces $Z \subset T \times E$ which are flat over $T$ and have 0 -dimensional fibres of constant lenght $n$. The Douady functor $\mathcal{D}^{n}$ is represented by a complex space $D^{n}(S \times E / S)$ over $S$, see [57]. For a point $s \in S, \mathcal{D}^{n}(\{s\})$ is the set of 0-dimensional subspaces of length $n$ and can be identified with the symmetric product $\operatorname{Sym}^{n}(E)$ because it is well known that the Hilbert-Chow morphism, in our case the DouadyBarlet morphism, $\mathcal{D}^{n}(\{s\}) \rightarrow\{s\} \times \operatorname{Sym}^{n}(E)$ is an isomorphism for the smooth curve $E$, see [5] Ch.V. It is then easy to show that also the relative Douady-Barlet morphism $D^{n}(S \times E / S) \rightarrow S \times \operatorname{Sym}^{n}(E)$ is an isomorphism. This implies that for any complex space $T$ over $S$ there is bijection

$$
\mathcal{D}^{n}(T) \stackrel{\sim}{\rightarrow} \operatorname{Hom}_{S}\left(T, S \times \operatorname{Sym}^{n}(E)\right) .
$$

One should note here that the behavior of families of cycles is more difficult to describe than of those for the Douady space.

Let now $\mathcal{D}_{r}^{n}$ resp. $\mathcal{M}_{X}(n, 0)_{r}$ be the restriction of the functors $\mathcal{D}^{n}$ and $\mathcal{M}_{X}(n, 0)$ to the category $(A n r / S)$ of reduced complex analytic spaces. By the Lemmas 2 and 3 the spectral covers give rise to a transformation of functors

$$
\mathcal{M}_{X}(n, 0)_{r} \stackrel{\gamma}{\rightarrow} \mathcal{D}_{r}^{n} \simeq \operatorname{Hom}_{S}\left(-, S \times \operatorname{Sym}^{n}(E)\right),
$$

where for a reduced space $T$ over $S$ and for a class $\left[\mathcal{F}_{T}\right]$ in $\mathcal{M}_{X}(n, 0)(T)$ we have $\gamma(T)\left(\mathcal{F}_{T}\right)=C\left(\mathcal{F}_{T}\right)$. Note that by flatness and compatibility with restriction to fibres, $C\left(\mathcal{F}_{T}\right)$ depends only on the equivalence class of $\mathcal{F}_{T}$. We are now able to present the theorem which generalises Theorem 8, see [26]. 
Theorem 11. The spectral cover induces a transformation of functors $\gamma: \mathcal{M}_{X}(n, 0)_{r} \rightarrow \operatorname{Hom}_{S}\left(-, S \times \operatorname{Sym}^{n}(E)\right)$ with the following properties.

(i) The functor $\mathcal{M}_{X}(n, 0)_{r}$ is corepresented by $S \times \operatorname{Sym}^{n}(E)$ via the transformation $\gamma$,

(ii) For any point $s \in S$ the induced map $M_{X_{s}}(n, 0) \rightarrow \operatorname{Sym}^{n}(E)$ is bijective. $S$.

(iii) The map $\gamma(T)$ is injective for any reduced complex space $T$ over

(iv) $\mathcal{M}_{X}(n, 0)_{r}$ is locally representable by $S \times \operatorname{Sym}^{n}(E)$, i.e. if $U \subset S$ is a trivializing open subset for $X$ and $T$ is a complex space over $U$, then $\gamma(T)$ is bijective.

The proof of the next result is based also on the spectral cover; see $[27]$.

Theorem 12. Consider an elliptic principal bundle $X \stackrel{\pi}{\rightarrow} S$ over a surface $S$, with at least one non-zero characteristic (Chern) class and with invariant $\epsilon=0$. If $S$ has no curves, then every rank-2 irreducible vector bundle $V$ on $X$ is a pull-back from $S$ up to a twist by a line bundle.

When $S$ is a projective manifold, a similar result was obtained by Verbitsky; see [63].

\section{REFERENCES}

[1] M. Aprodu, V. Brinzanescu, M. Toma, Holomorphic vector bundles on primary Kodaira surfaces, Math. Z. 242 (2002), 63-73.

[2] M. Aprodu, R. Moraru, M. Toma, Two-dimensional moduli spaces of vector bundles over Kodaira surfaces, Advances in Math. 231 (3-4) (2012), 1202-1215.

[3] M.F. Atiyah, Vector bundles over an elliptic curve, Proc.London Math.Soc. (3) 7, (1957), 414-452.

[4] C. Banica, J. Le Potier, Sur l'existence des fibrés vectoriels holomorphes sur les surfaces non-algebriques, J. Reine Angew. Math. 378 (1987), 1-31.

[5] D.Barlet, espaces analytique reduit des cycles analytiques complexes compact d'un espace analytique complexe de dimension finie, in Seminaire Norguet 1974-1975, Springer LNM 482, 1975.

[6] C. Bartocci, U. Bruzzo, D. Hernandez Ruiperez, Fourier-Mukai and Nahm transforms in geometry and mathematical physics, Progress in Math. 276, Birkhäuser, 2009.

[7] C. Bartocci, U.Bruzzo, D.Hernandez Ruiperez, J.M. Porras, Mirror symmetry on K3 surfaces via Fourier-Mukai transform, Comm.Math. Phys. 195 (1998) 79-93.

[8] K. Becker,M. Becker, K.Dasgupta, P.S. Green, Compactifications of heterotic theory on non-Kähler complex manifolds:I, JHEP 0304007 (2003).

[9] O. Ben-Bassat, Twisted derived equivalences, Trans. Amer. Math. Soc. 361 no.10 (2009), 5469-5504.

[10] A. Borel, A Spectral Sequence for Complex Analytic Bundles, Appendix II to F.Hirzebruch, Topological Methods in Algebraic Geometry, Third Edition, Springer-Verlag, 1966. 
[11] P. J. Braam, J. Hurtubise, Instantons on Hopf surfaces and monopoles on solid tori, J. Reine Angew. Math. 400 (1989), 146-172.

[12] T. Bridgeland, Equivalence of triangulated categories and Fourier-Mukai transforms, Bull. London Math.Soc. 31 no.1 (1999), 25-34.

[13] T. Bridgeland, Fourier-Mukai transforms for Elliptic Surfaces, J. Reine Angew. Math. 498 (1998), 115-133.

[14] T. Bridgeland, A. Maciocia, Fourier-Mukai Transforms for K3 and Elliptic Fibrations, J. Algebraic Geom. 11 (2002), 629-657.

[15] V. Brînzănescu, Holomorphic vector bundles over compact complex surfaces, Lecture Notes in Mathematics, 1624. Springer-Verlag, Berlin, 1996.

[16] V. Brinzanescu, Neron-Severi group for non-algebraic elliptic surfaces I: elliptic bundle case, Manuscripta Math. 79 (1993), 187-195.

[17] V. Brinzanescu, Neron-Severi group for non-algebraic elliptic surfaces II: non-kählerian case, Manuscripta Math. 84 (1994), 415-420.

[18] V. Brinzanescu, The Picard group of a primary Kodaira surface, Math. Ann. 296 (1993), 725-738.

[19] V. Brinzanescu, A simple proof of a Bogomolov type inequality in the case of non-algebraic surfaces, Rev. Roumaine Math. Pures Appl. 38 7-8 (1993), 631-633.

[20] V. Brinzanescu, P. Flondor, Holomorphic 2-vector bundles on nonalgebraic 2-tori, J. Reine Angew. Math. 363 (1985), 47-58.

[21] V. Brinzanescu, P. Flondor, Simple filtrable 2-vector bundles on complex surfaces without divisors, Rev. Roumaine Math. Pures Appl. 31 (1986), 507-512.

[22] V. Brinzanescu, R. Moraru, Holomorphic Rank-2 Vector Bundles on NonKähler Elliptic Surfaces Ann. Inst. Fourier, Grenoble 55, 5 (2005), 16591683.

[23] V. Brinzanescu, R. Moraru, Twisted Fourier-Mukai Transforms and Bundles on Non-Kähler Elliptic Surfaces, Math.Res.Lett. 13(2006), 501-514.

[24] V. Brinzanescu, R. Moraru, Stable Bundles on Non-Kähler Elliptic Surfaces, Comm. Math. Phys. 254(2005) pp.565-580.

[25] V. Brinzanescu, K. Ueno, Neron-Severi group for torus quasi-bundles over curves, Moduli of vector bundles (Sanda, 1994; Kyoto, 1994), 11-32, Lecture Notes in Pure and Appl. Math., 179, Dekker, New York, 1996.

[26] V. Brinzanescu, A. D. Halanay, G. Trautmann, it Vector bundles on nonKähler elliptic principal bundles Ann. Inst. Fourier, Grenoble 63, no. 3 (2013), 1033-1054.

[27] V. Brinzanescu, V. Vuletescu, Irreducible vector bundles on some elliptic non-Kähler threefolds, Journal of Geometry and Physics 91 (2015), 141145 .

[28] N. P. Buchdahl, Hermitian-Einstein connections and stable vector bundles over compact complex surfaces, Math. Ann. 280 (1988), 625-648.

[29] I. Burban, B. Kreussler, On a relative Fourier-Mukai transform on genus one fibrations, Manuscripta Math. 120 no.3 (2006), 283-306.

[30] A. Căldăraru, Derived Categories of Twisted Sheaves on Calabi-Yau Manifolds, Ph.D. thesis, Cornell University, May 2000.

[31] A. Căldăraru, Derived Categories of twisted sheaves on elliptic threefolds, J.Reine Angew. Math. 544(2002), 161-179.

[32] A.Căldăraru, J.Distler, S. Hellerman, T.Pantev, E. Sharpe, NonBirational Twisted Derived Equivalences in Abelian GLSMs, Comm. Math. Physics, 294 no.3 (2010), 605-645. 
[33] G.L. Cardoso, G. Curio, G. Dall'Agata, D. Lüst, P. Manousselis, G. Zoupanos, Non-Kähler string backgrounds and their five torsion classes, Nucl. Phys. B 652 (2003), 5-34.

[34] Pierre Deligne, Théorème de Lefschetz et critères de dégenérecence de suites spectrales, Publ. Mth. I.H.E.S. 35(1968), 107-126.

[35] R. Donagi, Principal bundles on elliptic fibrations, Asian J. Math. 1(1997), 214-223.

[36] R. Donagi, Spectral covers, Current topics in complex algebraic geometry (Berkeley, CA, 1992/93), 65-86, Math. Sci. Res. Inst. Publ. 28, Cambridge Univ. Press, Cambridge, 1995.

[37] R. Donagi, T. Pantev, Torus fibrations, gerbs, and duality, With an appendix by Dmitry Arinkin, Mem. Amer. Math. Soc. 193 (2008), no.901.

[38] A. Douady, Flatness and Privilege, Enseign. Math. 14(1968), 47-74.

[39] D. Eisenbud, Commutative Algebra with a View toward Algebraic Geometry, Springer-Verlag, 1995.

[40] G. Elencwajg, O. Forster Vector bundles on manifolds without divisors and a theorem of deformations, Ann. Inst. Fourier, Grenoble 32, 4 (1982), 25-51.

[41] R. Friedman, Rank two vector bundles over regular elliptic surfaces, Invent. Math., 96 (1989),283-332.

[42] R. Friedman, J. W. Morgan, E. Witten, Vector bundles over elliptic fibrations, J. Algebraic Geometry 8 (2) (1999), 279-401.

[43] P. Gauduchon, La 1-forme de torsion d'une varieté hermitienne compacte, Math. Ann. 267 (40) (1984), 495-518.

[44] E. Goldstein, S.Prokushkin, Geometric model for complex non-Kähler manifolds with SU(3) structure, Comm. Math. Phys. 251 no.1 (2004), 65-78.

[45] D. Hernández Ruiperéz, J.M. Muñoz Porras, Stable Sheaves on Elliptic Fibrations, J. Geom. Phys. 43 (2002), 163-183.

[46] N. Hitchin, Stable bundles and integrable systems, Duke Math. J. 54 (1) (1987), 91-114.

[47] Th. Höfer, Remarks on torus principal bundles, J.Math.Univ.Kyoto, 33 (1) (1993), 227-259.

[48] D. Huybrechts, Fourier-Mukai transforms in Algebraic geometry, Clarendon Press Oxford 2006.

[49] D. Huybrechts, M. Lehn, The geometry of moduli spaces of sheaves, Aspects in Mathematics E31, Vieweg-Verlag 1997.

[50] A.Kapustin, D.Orlov, Vertex algebras, mirror symmetry and D-branes: The case of complex tori, Comm.Math.Phys. 233 (2003), 79-136.

[51] K. Kodaira, On the structure of compact complex analytic surfaces I, Amer. J. Math. 86 (1964), 751-798.

[52] M. Lübke, A. Teleman, The Kobayashi-Hitchin correspondence, River Edge, NJ: World Scientific Publishing Co. Inc. 1995.

[53] R. Moraru, Integrable systems associated to a Hopf surface, Canad. J. Math. 55 (3) (2003), 609-635.

[54] S. Mukai, Duality between $D(X)$ and $D(\hat{X})$ with application to Picard sheaves, Nagoya Math. J., Vol. 81 (1981), 153-175.

[55] D. Mumford, Prym varieties I, Contributions to Analysis. Acad. Press, 1974, 325-350.

[56] D.Orlov, Derived categories of coherent sheaves and equivalences between them, Uspekhi Mat. Nauk, 351 (3) (2003), 89-172. 
[57] G.Pourcin, Theoreme de Douady au-dessus de S, Ann. Scuola Norm.Sup.Pisa 22 (1969), 451-459.

[58] H. W. Schuster, Locally free resolutions of coherent sheaves on surfaces, J. Reine Angew. Math. 337, (1982), 159-165.

[59] A. Teleman, Moduli spaces of stable bundles on non-Kähler elliptic fibre bundles over curves, Expo. Math. 16 (1998), 193-248.

[60] M. Toma, On the existence of simple reducible vector bundles on complex surfaces of algebraic dimension zero, Publ. R.I.M.S. Kyoto Univ. 27 (1991), 533-550.

[61] L. W.Tu, Semistable bundles over an Elliptic Curve, Adv. in Math. 98 (1993), 1-26.

[62] C. Voisin, A counterexample to the Hodge conjecture extended to Kähler varieties, Int. Math. Res. Not. 20 (2002), 1057-1075.

[63] M. Verbitsky, Stable bundles on positive principal elliptic fibrations, Math. Res. Lett. 12 (2-3) (2005), 251-264.

[64] V. Vuletescu, Existence of stable vector bundles on non-Kähler surfaces, C. R. Acad. Sci. Paris, Ser I 321 (5) (1995), 591-593.

[65] V. Vuletescu, Vector bundles over surfaces, Ph. D. thesis, Bucharest 1995.

[66] V. Vuletescu, Examples of coherent sheaves with no resolution by locally free sheaves in dimension 3, C. R. Acad. Sci. Paris, Ser. I 350 (2012), 411-412.

[67] V. Vuletescu, A restriction theorem for torsion-free sheaves on some elliptic manifolds, Bull. Math. Soc. Sci. Math. Roumanie (NS) 56 (104), (2), (2013), 237-242.

Institute of Mathematics "Simion Stoilow" of the Romanian AcadEmy, P.O. Box 1-764, 014700 Bucharest, Romania

E-mail address: Vasile.Brinzanescu@imar.ro 